\title{
Astrometric and photometric monitoring of GQ Lupi and its sub-stellar companion ${ }^{\star}$
}

\author{
R. Neuhäuser ${ }^{1}$, M. Mugrauer ${ }^{1}$, A. Seifahrt ${ }^{1}$, T. O. B. Schmidt ${ }^{1}$, and N. $\operatorname{Vogt}^{2,3}$ \\ 1 Astrophysikalisches Institut, Universität Jena, Schillergässchen 2-3, 07745 Jena, Germany \\ e-mail: rne@astro.uni-jena.de \\ 2 Departamento de Física y Astronomía, Universidad de Valparaíso, Avenida Gran Bretaña 1111, Valparaíso, Chile \\ 3 Instituto de Astronomía, Universidad Catolica del Norte, Avda. Angamos 0610, Antofagasta, Chile
}

Received 16 August 2007 / Accepted 4 December 2007

\section{ABSTRACT}

\begin{abstract}
Context. Neuhäuser et al. (2005, A\&A, 435, L13) presented direct imaging evidence for a sub-stellar companion to the young T Tauri star GQ Lupi. Common proper motion was highly significant, but no orbital motion was detected. Faint luminosity, low gravity, and a late-M/early-L spectral type indicated that the companion is either a planet or a brown dwarf.

Aims. We have monitored GQ Lupi and its companion in order to detect orbital and parallactic motion and variability in its brightness. We also search for closer and fainter companions.

Methods. We have taken six more images with the VLT Adaptive Optics instrument NACO from May 2005 to Feb. 2007, always with the same calibration binary from Hipparcos for both astrometric and photometric calibration. By adding up all the images taken so far, we search for additional companions.

Results. The position of GQ Lupi A and its companion compared to a nearby non-moving background object varies as expected for parallactic motion by about one pixel $(2 \cdot \pi$ with parallax $\pi)$. We could not find evidence for variability of the GQ Lupi companion in the $K_{\mathrm{s}}$-band (standard deviation being $\pm 0.08 \mathrm{mag}$ ), which may be due to large error bars. No additional companions are found with deep imaging.

Conclusions. There is now exceedingly high significance for common proper motion of GQ Lupi A and its companion. In addition, we see for the first time an indication for orbital motion ( 2 to 3 mas/yr decrease in separation, but no significant change in the position angle), consistent with a near edge-on or highly eccentric orbit. We measured the parallax for GQ Lupi A to be $\pi=6.4 \pm 1.9$ mas (i.e. $156 \pm 50 \mathrm{pc}$ ) and for the GQ Lupi companion to be $7.2 \pm 2.1$ mas (i.e. $139 \pm 45 \mathrm{pc}$ ), both consistent with being in the Lupus I cloud and bound to each other.
\end{abstract}

Key words. astrometry - stars: binaries: visual - stars: distances - stars: formation - stars: individual: GQ Lupi stars: pre-main sequence

\section{Introduction: GQ Lupi $A$ and its companion}

Based on three epochs of imaging data spanning five years, Neuhäuser et al. (2005, henceforth N05) presented evidence that the $\leq$ few Myr young T Tauri star GQ Lupi has a co-moving companion with $K_{\mathrm{s}} \simeq 13.1 \mathrm{mag}$ about $0.7^{\prime \prime}$ west $(\sim 100 \mathrm{AU}$ at $\sim 140 \mathrm{pc}$ ) of the primary star. A low-resolution spectrum gave evidence that it has a late-M to early-L spectral type. Temperature and luminosity can be used to estimate the mass via theoretical evolutionary models. They are, however, very uncertain at young ages (up to at least $10 \mathrm{Myr}$; Chabrier et al. 2005). Using the Wuchterl \& Tscharnuter (2003) model extended to planetary masses and few Myr of age, the GQ Lupi companion could be a 1 to $3 M_{\text {Jup }}$ object; whereas for the Tucson group models (Burrows et al. 1997), it is few to $\sim 30 M_{\text {Jup }}$; and for the Lyon group models (Baraffe et al. 2002), it is few to $\sim 40 M_{\text {Jup }}$. A comparison with the GAIA-1 model atmospheres indicated low gravity and, hence, a young age and very low mass (N05). Marois et al. (2007) re-analyzed archival HST and Subaru data to study the spectral energy distribution of GQ Lupi A and its companion,

* Based on observations collected at the European Southern Observatory, Chile in runs 073.C-0164, 075.C-0710C, 076.C-0339B, 077.C-0264B, 078.C-0552B, and 078.C-0535A. showing possible evidence for an excess in the $L$ - and $R$-bands (for the companion), possibly due to a disk and $\mathrm{H} \alpha$ emission; a fit of the data to the dusty GAIA model atmospheres confirmed the temperature and luminosity of the companion given in N05 and revised its radius to $0.38 \pm 0.05 R_{\odot}$, yielding a mass between 10 and $20 M_{\text {Jup }}$. McElwain et al. (2007) then obtained higher-resolution spectra $(R \simeq 2000)$ of the GQ Lupi companion in the $J$ - and $H$-bands with the integral field spectrograph OSIRIS at Keck and found a slightly higher temperature (M6L0) than in N05 (M9-L4), explained by the fact that $\mathrm{H}_{2}$ collisioninduced absorption is important for low gravity objects according to Kirkpatrick et al. (2006), but not considered in N05.

Higher-resolution spectra in the $J$-, $H$-, and $K$-bands taken with VLT/Sinfoni compared to GAIA-2 models could better constrain the parameters of the companion: the temperature is $2650 \pm 100 \mathrm{~K}$, the gravity $\log g=3.7 \pm 0.5 \mathrm{dex}$, and the radius $3.5_{-1.03}^{+1.50} R_{\text {Jup }}$ (Seifahrt et al. 2007a). Hence, its mass can be as low as few $M_{\text {Jup }}$, but also much higher. Comparing its parameters with 2M0535 A and B, where masses have been determined dynamically (an eclipsing double-lined binary brown dwarf in Orion, similar age as GQ Lupi, Stassun et al. 2006), could give an upper mass limit of $35 M_{\text {Jup }}$ (Seifahrt et al. 2007a). Thus, the companion to GQ Lupi can be regarded as a planet candidate according to the best guess value of its mass, which is at or 
Table 1. VLT/NACO observation log and astrometric calibration results from HIP 73357.

\begin{tabular}{|c|c|c|c|c|c|c|c|c|}
\hline Epoch & Date of & No. (a) & $\overline{\overline{F W H M}}$ & $\overline{\mathrm{Re}-}$ & \multicolumn{2}{|c|}{ Pixel scale in [mas/pixel] } & \multicolumn{2}{|c|}{ Detector orientation in $\left[{ }^{\circ}\right]$} \\
\hline year & observation & images & [mas] & mark & value and abs. err. & change and rel. err. & value and abs. err. & change and rel. err. \\
\hline 2004.48 & 25 Jun. 2004 & 27 & 64 & (b) & $13.23 \pm 0.05$ & (b) & $0.14 \pm 0.25$ & (b) \\
\hline 2005.40 & 27 May 2005 & 18 & 74 & & $13.240 \pm 0.050$ & $0 \pm 0.001$ & $0.21 \pm 0.33$ & $0 \pm 0.007$ \\
\hline 2006.14 & 22 Feb. 2006 & 15 & 66 & (c) & $13.238 \pm 0.053$ & $-0.002 \pm 0.003$ & $0.18 \pm 0.35$ & $-0.024 \pm 0.020$ \\
\hline 2006.38 & 20 May 2006 & 15 & 100 & & $13.233 \pm 0.055$ & $-0.007 \pm 0.006$ & $0.39 \pm 0.36$ & $0.184 \pm 0.028$ \\
\hline 2006.54 & 16 Jul. 2006 & 15 & 73 & & $13.236 \pm 0.055$ & $-0.004 \pm 0.005$ & $0.43 \pm 0.36$ & $0.221 \pm 0.031$ \\
\hline
\end{tabular}

Remarks: for each VLT/NACO observation, we list the pixel scale determined with its absolute error and the change in pixel scale since the first new observation (2005.4), always with the same astrometric standard star HIP 73357; same for detector orientation in the last two columns. (a) Each image consists of 200 exposures with $0.347 \mathrm{~s}$ each, for both GQ Lupi and the astrometric calibration HIP 73357 . (b) Results from 2004.48 as in N05, no relative errors available, because another astrometric calibration binary was observed. (c) GQ Lupi A in a non-linear regime (not saturated). (d) GQ Lupi A and HIP 73357 A in a non-linear regime (not saturated). Calibration (for 19 Feb. 2007 GQ Lupi data) obtained on 1 Mar. 2007 with HIP 73357, see footnote 1.

below the brown dwarf desert ( $\sim 30 M_{\text {Jup }}$; Grether \& Lineweaver 2006), proposed as the deviding line between planets and brown dwarfs. Also, it is possible that the true mass is below $13 \mathrm{M}_{\mathrm{Jup}}$, a more conservative upper mass limit for planets.

The large error in the luminosity of the companion, which is used for the mass estimate from evolutionary models, is mostly due to a large distance uncertainty, assuming that the object is in Lupus I ( $\pm 50 \mathrm{pc}, \mathrm{N} 05)$. Hence, a direct parallax measurement would yield a stronger contraint on luminosity and, hence, mass.

To finally confirm that the fainter object near GQ Lupi is really a bound companion (rather than, e.g., another member of the Lupus I cloud not orbiting GQ Lupi), one would need to see orbital motion.

For both measurements, parallax and orbital motion, we have monitored GQ Lupi and its companion from 2005 to 2007 by taking six new images with Adaptive Optics. In Sects. 2 and 3, we explain the observations, data reduction and astrometric results.

We also use the data to monitor the brightness of GQ Lupi A and its companion and search for photometric variability (see Sect. 4). We note that Seifahrt et al. (2007a) found emission lines in the near-infrared spectra of the companion, indicative of accretion, so some variability is expected. We then add up all imaging data thus far available to obtain a very deep and high-dynamic-range image to search for additional, fainter and/or closer companions (see Sect. 5). We note that both Debes \& Sigurdsson (2006) and Boss (2006) argued that if the GQ Lupi companion is a planet, it should have been moved to its current large separation ( $\sim 100 \mathrm{AU})$ from an originally closer orbit by an encounter with another more massive proto-planet, which may be detectable. We use the newly determined parameters to re-estimate some physical parameters of the GQ Lupi companion in Sect. 6 and summarize our results at the end.

\section{Observations with VLT/NACO}

We observed GQ Lupi A and its companion six times from May 2005 until Feb. 2007 in order to detect and monitor possible changes in separation, position angle, and brightness. See Table 1 for the observations log, where we also include the first VLT imaging observation from June 2004, which we re-reduced here.

All observations were done with the European Southern Observatory (ESO) Very Large Telescope (VLT) instrument Naos-Conica (NACO; Rousset et al. 2003) in the $K_{\mathrm{s}}$-band around
$2 \mu \mathrm{m}$ : 200 images (NDIT) with $0.347 \mathrm{~s}$ (DIT) each, the shortest possible integration time, all added up automatically to one frame (without moving the telescope). We then took 15 frames (NINT) with GQ Lupi on slightly different positions (jitter or dither technique), but 18 frames in May 2005 and 27 in June 2004. We always used the S13 camera (pixel scale roughly 13 mas/pixel) and the double-correlated read-out mode. For the astrometric calibration binary HIP 73357, observed within one hour $^{1}$ of the GQ Lupi observations with the same set-up, we took five frames, i.e., five jitter positions with $200 \times 0.347 \mathrm{~s}$ each (but six such images in May 2005 and four each in May and July 2006). For the raw data reduction, we subtracted a mean dark from all the science frames and flats, then devided by the mean normalized dark-subtracted flat and subtracted the mean background.

Compared to the data reduction for N05, we now use a new, improved IDL routine for the subtraction of the point spread function (PSF) of the bright GQ Lupi A, before measuring the companion. The new routine centers the bright star PSF with sub-pixel precision: after rotating the PSF of GQ Lupi A around its center 179 times in steps of 2 degrees, we subtract the rotated image from the original image, and then take the median of all resulting images. This gives the final PSF-subtracted image, which we use to measure the companion (for astrometry and photometry).

We re-reduced the GQ Lupi data from June 2004 (published in N05) and thereby obtained slightly better (larger) values for separation and brightness, consistent with N05 within the errors. The new values are given in Table 2 . The new values for GQ Lupi for 2004 use the same calibration results as in N05 for pixel scale and detector orientation (Table 1), since it was not necessary to re-reduce the 2004 calibration binary observation.

\section{Astrometry}

Since GQ Lupi has right ascension 15 h 49 m, we always placed one observation in May (2005 and 2006), when it is visible the whole night, two observations three months earlier (Feb. 2006 and 2007) and two more observations three months

\footnotetext{
1 In the Feb. 2007 service mode observation, HIP 73357 B was inside the field-of-view only in two out of five frames, so we could not use it properly. Instead, we used HIP 73357 observations done with the identical set-up a few nights later on 1 March 2007 (run 078.C-0535A), where 43 useful high- $S / N$ frames were taken $(80 \times 0.347 \mathrm{~s}$ each) by some of us. We used them to calibrate the 19 Feb. 2007 observations of GQ Lupi, because there was no intervention into NACO in the meantime, as confirmed with ESO.
} 
Table 2. Separation and PA between GQ Lupi and its companion.

\begin{tabular}{|c|c|c|c|c|c|c|c|c|}
\hline $\begin{array}{l}\text { Epoch } \\
\text { year }\end{array}$ & $\begin{array}{l}\text { Separation } \\
\text { (absolute) } \\
\text { [pixel] (a) }\end{array}$ & $\begin{array}{c}\text { Separation } \\
\text { (absolute) } \\
\text { [mas] (a) }\end{array}$ & $\begin{array}{c}\text { Sep. change } \\
\text { since 2005.4 } \\
\text { [mas] (b) }\end{array}$ & $\begin{array}{c}\text { Position Angle } \\
\text { (absolute) } \\
\text { [deg] (a) }\end{array}$ & $\begin{array}{c}\text { Change in PA } \\
\text { since 2005.4 } \\
\text { [deg] (b) }\end{array}$ & $\begin{array}{l}\text { Sign. } \\
\text { being } \\
\text { re PA }\end{array}$ & $\begin{array}{l}\text { gainst } \\
/ \text { g (d) } \\
\text { re sep }\end{array}$ & $\begin{array}{l}\text { Sign. for } \\
\text { orbital } \\
\text { motion (e) }\end{array}$ \\
\hline 2004.48 & $55.53 \pm 0.11$ & $734.7 \pm 3.1$ & (c) & $275.48 \pm 0.25$ & (c) & $3.2 \sigma$ & $4.5 \sigma$ & - \\
\hline 2005.40 & 13 & 735 & \pm 0.5 & 27 & $0 \pm 0$ & - & - & - \\
\hline 2005.60 & $55.34 \pm$ & $733.3 \pm$ & $-1.8 \pm 1.2$ & 275.8 & $-0.13=$ & $0.9 \sigma$ & $1.1 \sigma$ & $0.5 \sigma$ \\
\hline 2006.14 & $55.13 \pm 0.12$ & $729.8 \pm 3.3$ & $-5.3 \pm 1.0$ & $276.14 \pm 0.35$ & $0.14=$ & $2.4 \sigma$ & $4.7 \sigma$ & $4.2 \sigma$ \\
\hline 2006.38 & $55.27 \pm 0.15$ & $731.4 \pm 3.5$ & $-3.7 \pm 1.0$ & $276.06 \pm 0.38$ & $0.06 \pm 0.12$ & $3.9 \sigma$ & $5.2 \sigma$ & $2.6 \sigma$ \\
\hline 2006.54 & $55.40 \pm 0.30$ & $733.2 \pm 5.0$ & $-1.9 \pm 2.1$ & $276.26 \pm 0.68$ & $0.26 \pm 0.58$ & $4.1 \sigma$ & $4.0 \sigma$ & $0.0 \sigma$ \\
\hline 2007.13 & $55.14 \pm 0.42$ & $730.0 \pm 6.4$ & $-5.1 \pm 4.6$ & $276.04 \pm 0.63$ & $0.04 \pm 0.50$ & $5.0 \sigma$ & $3.1 \sigma$ & $0.5 \sigma$ \\
\hline
\end{tabular}

Remarks: PA is from north over east to south, after correcting for the detector orientation.

(a) Absolute errors; (b) relative errors; (c) no relative errors available, because another astrometric calibration binary was observed; (d) significance in Gaussian $\sigma$ against background (b/g) hypothesis for each epoch compared to the first new epoch (2005.4) regarding (re) PA (Fig. 1) and separation (Fig. 2); the background hypothesis is that the companion is a non-moving background object, in which case GQ Lupi A would move away due to its known proper motion. The significances compared from the three earlier observations from 1994 to 2002 are 5.4, 7.0, and 6.9 $\sigma$ for separation, respectively, and 7.6 and $8.3 \sigma$ for PA of 2005.4 compared to 1999 and 1994, respectively; (e) the significance for deviation of each separation value from the 2005.4 value is given in the last column (see Fig. 2b). It can be seen as evidence for orbital motion; (d, e) because the measurements are independent, we can add up the significances (properly) and obtain altogether very high significance against the background hypothesis and $\geq 5 \sigma$ for having detected orbital motion.

later (July/Aug. 2005 and 2006), when GQ Lupi is still visible at the end or start of the night, respectively, so that we can use the data not only for proper motion and photometric monitoring, but also for trying to determine the absolute or relative parallaxes of GQ Lupi A and its companion.

For the astrometric calibration, we observed the Hipparcos binary star HIP 73357 in each run. See the online Appendix for more information and Table 1 for the results.

\subsection{Astrometric data reduction on GQ Lupi}

One can combine all images taken within, e.g., one night by standard shift+add to obtain one image with very high $S / N$. We did this in N05, and have done the same for the six new observations from 2005 to 2007. In the final image, one can then determine the PSF photocenter of the bright star GQ Lupi A and, after PSF subtraction, also the PSF photocenter of the companion. This results in measurements of separation in pixels and the position angle (PA) of the companion relative to star A. The companion is always slightly north of west. The error for the separation include errors from the Gaussian centering on A and companion, as well as the error in the pixel scale determined; the error for the PA includes centering errors and the error in the north-south alignment of the detector during the observation. In Table 2, we list the separation in pixels and arcsec (computed with the pixel scales from Table 1) and also the PA in degrees, as corrected with the detector orientation given in Table 1 for each epoch.

To check the errors in separation and/or PA, we not only measured the photocenters of GQ Lupi and its companion in the one full final high- $S / N$ image after combining all images of one epoch (one night), but we also measured separation and PA in any of the 15 to 27 GQ Lupi images, as well as in bins of 3, 5, 7, and 9 GQ Lupi images. With bins of, e.g., $x$ images, we mean here the combination of $x$ individual images by shift+add, e.g., we can obtain three combined images with five individual images added up (or e.g. 5 combined images with 3 individual images added up). We can then determine separation (and PA) in any of the combined images. The weighted mean of those values is then the final measurement of separation (and PA) of that epoch, and the standard deviation is the precision of that measurement. We use the larger error values in Table 2 for the absolute errors. Since the calibration binary HIP 73357 has changed its PA significantly since 1991.25 , the calibration errors in PA are relatively large, so that they overwhelm real measurement errors.

We correct the separation measured in pixels and the PA measured for GQ Lupi and its companion with the calibration values given in Table 1. In Table 2, we list the values for separation and PA between GQ Lupi A and its companion. In Figs. 1 and 2, we plot the PA and separations versus time, as observed and listed in Table 2. The precision of the measurements decrease slightly from 2005 to 2006 and are worst in 2007, where we have strong reflection effects and a strong waffle structure (bad seeing, large $F W H M$ ). This decrease is at least partly due to the fact that the error in the astrometric calibration binary from its possible orbital motion since 1991.25 (Hipparcos) increases with time. The 2006 data for both GQ Lupi and HIP 73357 also have much lower $S / N$ than those in 2004 and 2005.

For the last seven NACO measurements, the mean in separation between GQ Lupi A and its companion is 732.1 \pm 2.1 mas and the mean PA is $275.98 \pm 0.25^{\circ}$, i.e., slightly north of west. This separation corresponds to $114 \pm 33 \mathrm{AU}$ at $156 \pm 50 \mathrm{pc}$, the distance to GQ Lupi A determined in Sect. 3.4.

\subsection{Proper motion and orbital motion}

Given the proper motion of GQ Lupi A (see Mugrauer \& Neuhäuser 2005), we now have exceedingly high significance against the background hypothesis (that the faint object is a nonmoving background object with negligible parallax; see Figs. 1 and 2 and Table 2). It is also very unlikely that both objects are independent members of the Lupus I cloud with similar proper motions (see Mugrauer \& Neuhäuser 2005).

For final confirmation that they are bound, one could, e.g., try to measure the parallaxes of both objects with high precision (not yet feasible) or observe curvature in the orbital motion (possible after a few more decades).

For $\sim 114$ AU projected separation (see Sect. 3.4) and a circular orbit, one would expect $\sim 1372$ years orbital period and, hence, up to a few mas/yr orbital motion (e.g. up to $6.7 \mathrm{mas} / \mathrm{yr}$ for an eccentricity $e=0.5$ for edge-on inclination), and less than $0.3^{\circ} \mathrm{PA}$ change for $e=0\left(\leq 0.82^{\circ} \mathrm{PA}\right.$ for $e=0.5$ for poleon orbit). The possible changes in separation and PA seen in Figs. 1 and 2 are smaller than these maxima, hence within the expectation. 

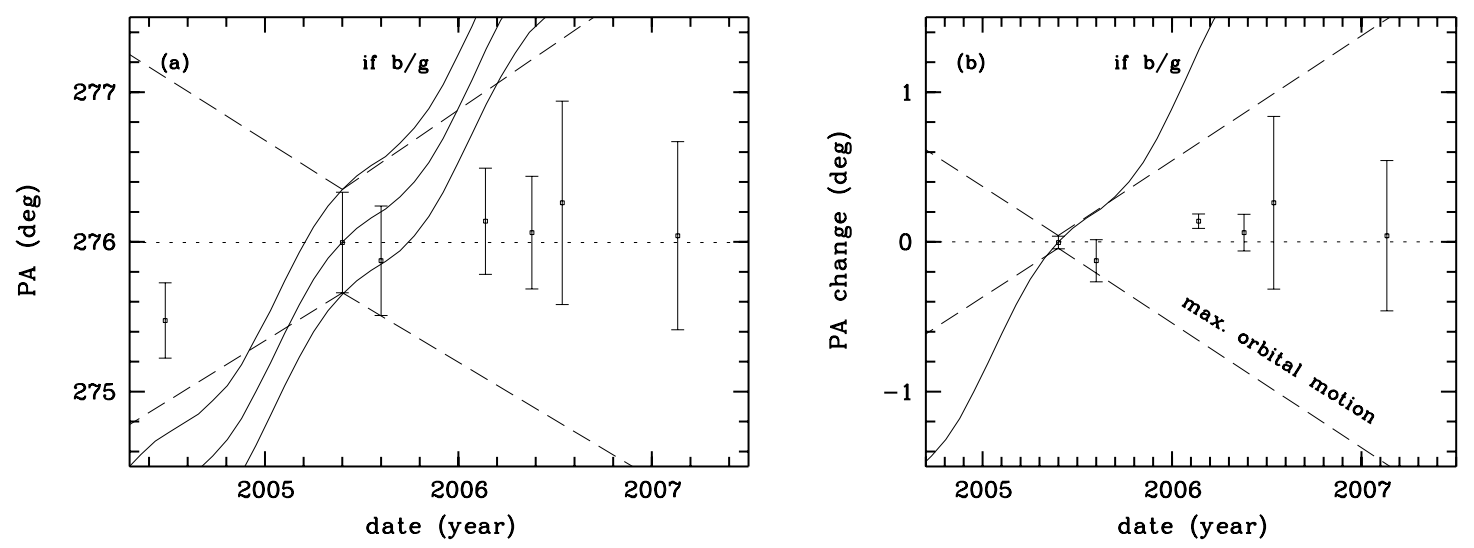

Fig. 1. PA of the companion relative to GQ Lupi A versus time. a) Absolute PA values with their absolute errors for all NACO observations (Table 2). b) Change in PA since May 2005 with relative errors, the error includes orbital motion of the HIP 73357 calibration binary since 2005.4 only (not since 1991.25 as in the left panel). We also show the data point at the first new epoch 2005.4, set to 0.0, with its error bar just from the Gaussian centering fit. For both $\mathbf{a}$ ) and b), the straight dotted line is for constant PA, as expected if both objects are bound. To allow for orbital motion, the maximum change in PA (if pole-on orbital plane) is indicated by dashed lines. The data stay constant or vary slowly within those limits. There is no strong evidence for orbital motion in PA; the first data point from 2004 should have lower weight here, because it was obtained with different astrometric calibration binaries than in 2005 to 2007. The full wobbled lines with strong positive slope indicate the change in PA, if the companion was background ( \pm expected errors from errors in proper motion and parallax in panel a); this hypothesis can be rejected with large significance, because the data points strongly deviate from this hypothesis.

Orbital motion is detectable as deviation among the separation and/or PA values. The data points from Feb. and May 2006 in the lower left panel (Fig. 2b) are deviant from the May 2005 value (drawn as the full line for constant separation) by 4.2 and $2.6 \sigma$, respectively, so that we have a formal significance of 4.2 and $2.6 \sigma$ for detection of orbital motion. We observe a possible small (constant, linear) change in separation, namely a decrease by $\sim 2$ to $3 \mathrm{mas} / \mathrm{yr}$ (best fit to the last six data points, see Fig. 2), in particular a better fit compared to constant separation (732.1 mas being the mean) regarding rms scatter, which has a reduced $\chi^{2}=0.320$, and also a better fit compared to different parallaxes for GQ Lupi A and its companion. We detect no significant change in PA. This would be consistent with an orbital plane that is more edge-on than pole-on or with a highly eccentric orbit, as already argued by Janson et al. (2006) based on the data from 1994 until 2004 only.

The maximum motion of the GQ Lupi companion of $\sim 2$ to 3 mas/yr or $\sim 2 \mathrm{~km} \mathrm{~s}^{-1}$ in one dimension is significantly smaller than the expected escape velocity from the GQ Lupi A system, which is $5 \pm 2 \mathrm{~km} \mathrm{~s}^{-1}$, as already concluded with previous data in Mugrauer \& Neuhäuser (2005).

From the measured projected equatorial rotational velocity $v \cdot \sin i=6.8 \pm 0.4 \mathrm{~km} \mathrm{~s}^{-1}$, the newly determined rotation period (8.45 \pm 0.2 days), and the luminosity and temperature of GQ Lupi A, Broeg et al. (2007) estimated the inclination of the star (GQ Lupi A) to be $27 \pm 5^{\circ}$ degrees, which is more pole-on than edge-on. Unless the orbit of the companion is highly eccentric, this may indicate that the stellar equator and the companion have different planes. Normally, one would expect a planet to form in or nearly in the equatorial plane of the star. If the GQ Lupi companion is a planet, however, it probably has not formed at its current wide separation, but further inward, and was then moved to a larger separation by an encounter with another, more massive inner protoplanet (Debes \& Sigurdsson 2006; Boss 2006). An encounter with another star could have resulted in a dynamical perturbation of the forming GQ Lupi (system) resulting in a binary with large mass ratio, i.e., a companion forming as a brown dwarf (embryo) by fragmentation, and/or would also have increased the orbital inclination and/or eccentricity of the (forming) companion significantly.

Deviations of the separation and PA values from being constant could also be due to other reasons:

(i) If either GQ Lupi A or b were an unresolved multiple, one would expect a periodic variation in the separation. If, e.g., GQ Lupi A was an unresolved close binary made up of two stars with different brightness, its photocenter would show periodic variations according to the Keplerian motion of the two stars around its common center-of-mass. Then, the apparent separation between this photocenter and GQ Lupi b would also vary with the same period. Quantitatively, this effect depends on the separation between the two stars (of this hypothetical binary) and their brightness difference. If the observed change in separation between GQ Lupi A and b was due to this effect, the time-scale is short, of the order of months to few years, so the separation between the two stars would be limited to a few AU. Even for large mass and brightness differences between the two stars, the effect would then be limited to about 0.14 mas/yr. The observed effect is much larger $(5.3 \pm 1.1 \mathrm{mas} / \mathrm{yr}$, comparing the separations in 2005.40 and 2006.14), hence cannot be explained by this effect.

(ii) GQ Lupi A and its apparent companion b do not orbit each other, but have slightly different proper motions. This possibility has already been discussed in Mugrauer \& Neuhäuser (2005). Since signatures of accretion are detected in both objects (A and companion; Seifahrt et al. 2007a), both are young and, hence, are most likely members of the Lupus I could. The velocity dispersion in star forming regions like Lupus is only a few mas/yr, the same order of magnitude as the difference in proper motion observed here. The probability, however, to find two young objects within $\sim 0.7^{\prime \prime}$ with almost exactly the same proper motion (which is different from the mean Lupus proper motion, Mugrauer \& Neuhäuser 2005), and at the same time not orbiting each other, is exceedingly small. We could also show (in Fig. 2) that the assumption that the two objects are unbound at 

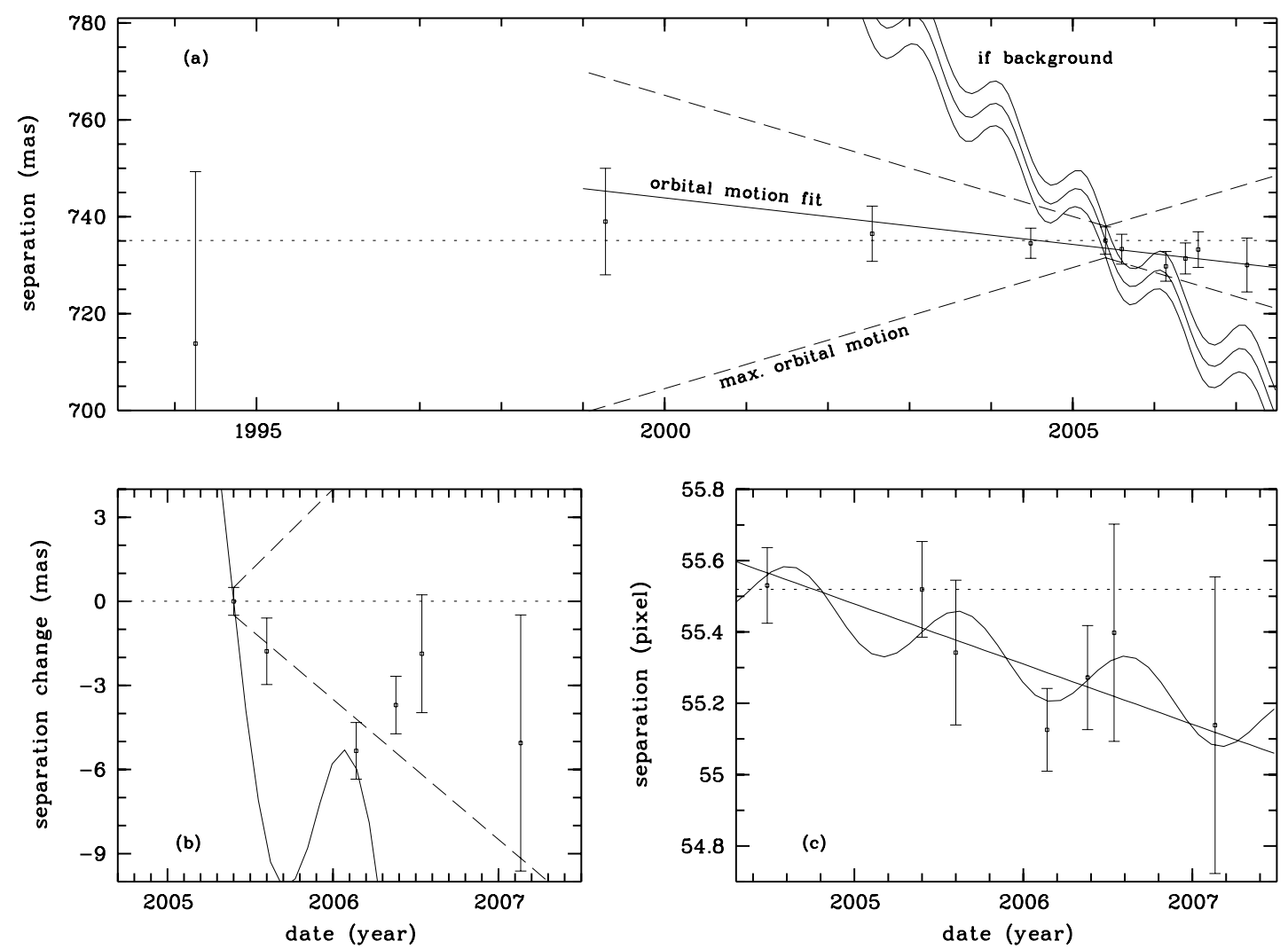

Fig. 2. Separation between GQ Lupi A and its companion plotted versus time. Data are from Janson et al. (2006) for 1994, N05 for 2002, and this paper for the rest. Constant separation is indicated as a straight dotted line (through 2005.4 value), separation changes due to maximum expected orbital motion are indicated by dashed lines. All values are well within the expectations for orbital motion. a) Absolute values from 1994 to 2007. Here, we also indicate as a wobbled line with strongly decreasing values the expected separation change for the background hypothesis, computed from the known GQ Lupi A proper motion and parallax and assuming that the faint object has negligible proper and parallactic motion ( \pm errors as expected from proper motion and parallax errors as additional wobbled lines); the data are strongly deviant from the background hypothesis. The full line from 1999 to 2007 with smaller negative slope is the best fit for the orbital motion ( 2 to 3 mas/yr separation decrease from a linear fit to the last six data points), see text. b) Separation change since May 2005 with relative errors (including the data point from the first new epoch 2005.4, set to 0.0, with error bar just from Gaussian centering fits): the data points from Feb. and May 2006 are deviant from the dashed constant line (for constant separation) by 4.2 and $2.6 \sigma$, respectively, significance for orbital motion. The wobbled line again is for the background hypothesis. c) Separation in pixel with absolute errors (since June 2004). The full line with negative slope is our best fit for orbital motion as in a); this fit has a reduced $\chi^{2}$ of 0.250 with 5 degrees of freedom and, hence, a probability of 0.94 (free fit parameters being starting separation and slope of decreasing separation). The wobbled line here is for assuming that GQ Lupi A and the faint object would have different parallaxes (different by 1.5 mas as best fit); this fit has a reduced $\chi^{2}$ of 0.221 with 4 degrees of freedom and, hence, a probability of only 0.92 (free fit parameters being starting separation, slope of decreasing separation, and difference in parallaxes between GQ Lupi A and its co-moving companion). Hence, the full line (for identical parallaxes for GQ Lupi A and companion) is a better fit than the wobbled line. This gives evidence against the hypothesis that the deviations in separations observed, in particular in 2005.6 and 2006.4 (compared to 2005.4), are due to different parallaxes for GQ Lupi A and its companion.

different parallaxes gives a worse fit (to the observed separation changes) than the assumption that they are at the same distance orbiting each other.

(iii) The apparent effect could have been introduced by incorrect calibration. We have corrected each original separation and PA measurement for GQ Lupi by the individual calibration results from HIP 73357. It could be that pixel scale and/or detector orientation, however, do not change with time, therefore the fact that we measure different values is not real, but just within the noise. However, when plotting separations in pixels (Fig. 2c) or the measured original uncorrected PA in degrees (not shown, but see Table 2), i.e., both without astrometric calibration correction versus time, we still see the same effect, a small decrease in separation and no effect in PA (actually an even larger scatter in PA, so that the individual correction is most likely needed).
Hence, orbital motion is the most likely cause for the small (linear) variation in the separation; quantitatively, the variation is within expectation.

\subsection{The parallax of GQ Lupi}

The NACO observations were also used to determine the parallax of GQ Lupi A and its companion. The largest error source in the luminosity of the companion and, hence, its mass determination is the uncertain distance (90 to $190 \mathrm{pc}$ for Lupus I, N05). In the north-west corner of the small (S13) NACO fieldof-view (FoV), there is an additional star detected in all NACO images, about 6.4" NW of GQ Lupi A. As any faint object near a bright star, we have to see it as a companion candidate, which we call GQ Lupi/cc2 ( $c$ f for companion candidate, GQ Lupi b formerly was GQ Lupi/cc1). See Table 3 for the separation of this object (relative to both GQ Lupi A and its confirmed 
Table 3. Astrometry on background star cc2 with VLT/NACO.

\begin{tabular}{l|cc|cc}
\hline \hline $\begin{array}{l}\text { Epoch } \\
\text { year }\end{array}$ & \multicolumn{2}{|c}{$\begin{array}{c}\text { Separation between GQ Lupi A and cc2 } \\
\Delta \text { RA }\left[{ }^{\prime \prime}\right]\end{array}$} & $\Delta$ Dec $\left[{ }^{\prime \prime}\right]$ & $\Delta$ Separation between GQ Lupi b and cc2 \\
\hline 2004.48 & $4.3265 \pm 0.0164$ & $4.6473 \pm 0.0175$ & $3.5953 \pm 0.0137$ & $4.5775 \pm 0.0173$ \\
2005.40 & $4.3120 \pm 0.0164$ & $4.6739 \pm 0.0177$ & $3.5812 \pm 0.0136$ & $4.5969 \pm 0.0174$ \\
2005.60 & $4.2977 \pm 0.0163$ & $4.6715 \pm 0.0177$ & $3.5703 \pm 0.0135$ & $4.5956 \pm 0.0174$ \\
2006.14 & $4.3041 \pm 0.0163$ & $4.6909 \pm 0.0178$ & $3.5786 \pm 0.0136$ & $4.6134 \pm 0.0175$ \\
2006.54 & $4.2888 \pm 0.0163$ & $4.6965 \pm 0.0179$ & $3.5590 \pm 0.0136$ & $4.6272 \pm 0.0179$ \\
\hline
\end{tabular}

companion b) corrected for pixel scale and detector orientation; the largest contribution to the error budget is due to the large separation in pixels (several hundred pixels) between the object cc2 and the two components of the GQ Lupi system, to be multiplied by the error in the pixel scale, and then taken into account in the proper error propagation. The data from 2006.38 and 2007.13 have the largest errors, which are due to the poor FWHM at these epochs (see Table 1), cc2 is only marginally detected at those two epochs. We therefore exclude those two data points in the parallax determination (Table 3, Figs. 3 and 4), as they do not give any further constraints (they are consistent with the solution given).

We can first verify that GQ Lupi/cc2 is a background star, Figs. 3 and 4, because separation between this object and GQ Lupi A (and also the separation between cc2 and the GQ Lupi companion) in both right ascension and declination change as expected from the known proper motion of GQ Lupi A. When assuming that cc2 does not move, then we obtain as proper motion for GQ Lupi A $\mu_{\alpha}=-16.8 \pm 4.2 \mathrm{mas} / \mathrm{yr}$ and $\mu_{\delta}=-24.3 \pm 2.2 \mathrm{mas} / \mathrm{yr}$, and for the known co-moving GQ Lupi companion we get $\mu_{\alpha}=-15.2 \pm 4.6 \mathrm{mas} / \mathrm{yr}$ and $\mu_{\delta}=-23.7 \pm 2.7 \mathrm{mar} / \mathrm{yr}^{2}$. Hence, the (proper and parallactic) motion of cc2 is negligible, therefore we can use it for measuring the parallax of GQ Lupi A and its companion from the scatter in Figs. 3 and 4, namely deviations from a linear motion. This background object (GQ Lupi/cc2) is listed in USNO-B1 as object 00543-0373323 with $B_{2}=19.28 \mathrm{mag}, R_{2}=17.77 \mathrm{mag}$, and $I=$ 15.94 mag; it is also listed in NOMAD as object 0543-0380757 with $B=19.53 \mathrm{mag}$. We have determined $K_{\mathrm{s}}=15.01 \pm 0.33 \mathrm{mag}$ (see Table 4). Hence, it is probably a mid- to late-K dwarf (or giant) star with weak extinction. If it is on the main sequence, it has a distance of 800 to $1700 \mathrm{pc}$, i.e., a parallax of $1.0 \pm 0.3$ mas (even smaller if a giant). Its own proper motion is negligible, as seen above.

The timing of our NACO observations in Feb., May, and Aug. 2005 to 2007 was chosen, because the offset in the separation due to parallactic motion is maximal at these times.

The wobble remaining in the motion of GQ Lupi A (and its companion) after subtracting the proper motion is then the parallactic motion. In Figs. 3 and 4, we show the data points (difference in position between GQ Lupi A and companion, respectively, and the third star cc2), and our best fit giving the parallax of GQ Lupi A to be $7.4 \pm 1.9$ mas and the parallax of the GQ Lupi companion to be $8.2 \pm 2.1$ mas (full error propagation). For the fit to the GQ Lupi A data, the wobbled curve with a non-zero parallax has a reduced $\chi^{2}=0.036$ for RA and $\chi^{2}=0.011$ for Dec, while a linear fit (decreasing separation, no parallax) has $\chi^{2}=0.167$ for RA and 0.037 for Dec. Hence, our fit with $\pi=7.4 \pm 1.9$ mas is better. For the fit to

\footnotetext{
2 These two proper motions are not only consistent with each other, but also consistent with the published proper motion of GQ Lupi A being $\mu_{\alpha}=-19.15 \pm 1.67 \mathrm{mas} / \mathrm{yr}$ and $\mu_{\delta}=-21.06 \pm 1.69 \mathrm{mas} / \mathrm{yr}$ (Mugrauer \& Neuhäuser 2005).
}
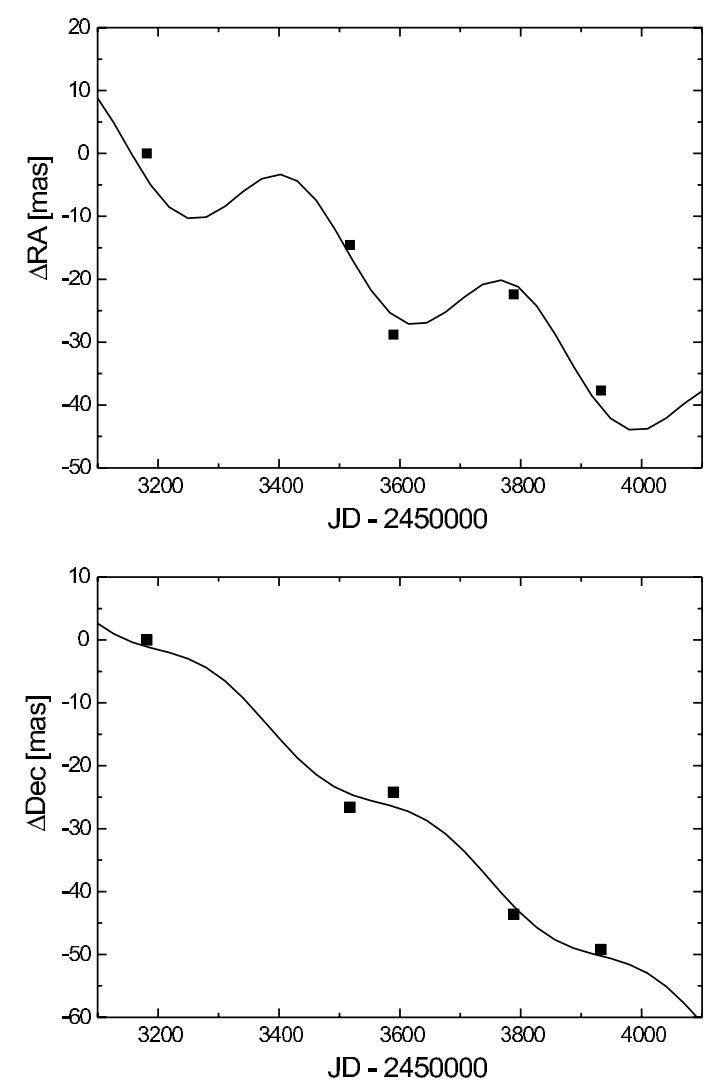

Fig. 3. Proper and parallactic motion of GQ Lupi A in right ascension (top) and declination (bottom) relative to the background object GQ Lupi/cc2. Changes in separations in mas (since 2004.5) are plotted versus observing epoch as Julian date JD. We also show our best fit yielding a parallactic wobble of $7.4 \pm 1.9$ mas, from which we have to subtract the probable parallax of the background object cc 2 $(1.0 \pm 0.3$ mas, see text), thus obtaining $6.4 \pm 1.9$ mas or $156 \pm 50 \mathrm{pc}$ for GQ Lupi A.

the data on the GQ Lupi companion, the wobbled curve with a non-zero parallax has a reduced $\chi^{2}=0.022$ (for RA) and 0.039 (for Dec), while a linear fit (decreasing separation, no parallax) has $\chi^{2}=0.288$ (for RA) and 0.058 (for Dec). Hence, again, our fit with $\pi=8.2 \pm 2.1$ mas is better.

The two values for GQ Lupi A and its companion are consistent with each other. For GQ Lupi A, the parallax corresponds to $\sim 135 \mathrm{pc}$ (110 to $180 \mathrm{pc}$ ) or roughly $135 \pm 30 \mathrm{pc}$, i.e., more precise than the previous distance estimate for the Lupus I cloud including GQ Lupi being $140 \pm 50$ pc (N05). For the GQ Lupi companion, the parallax corresponds to $\sim 122 \mathrm{pc}(100$ to $160 \mathrm{pc})$.

Our parallax measurements are based on only one comparison star (cc2), which could move itself (proper and/or parallactic motion). The above values are correct when assuming that this background object has negligible proper and parallactic motion. The fact that we obtain the correct GQ Lupi proper motion when 

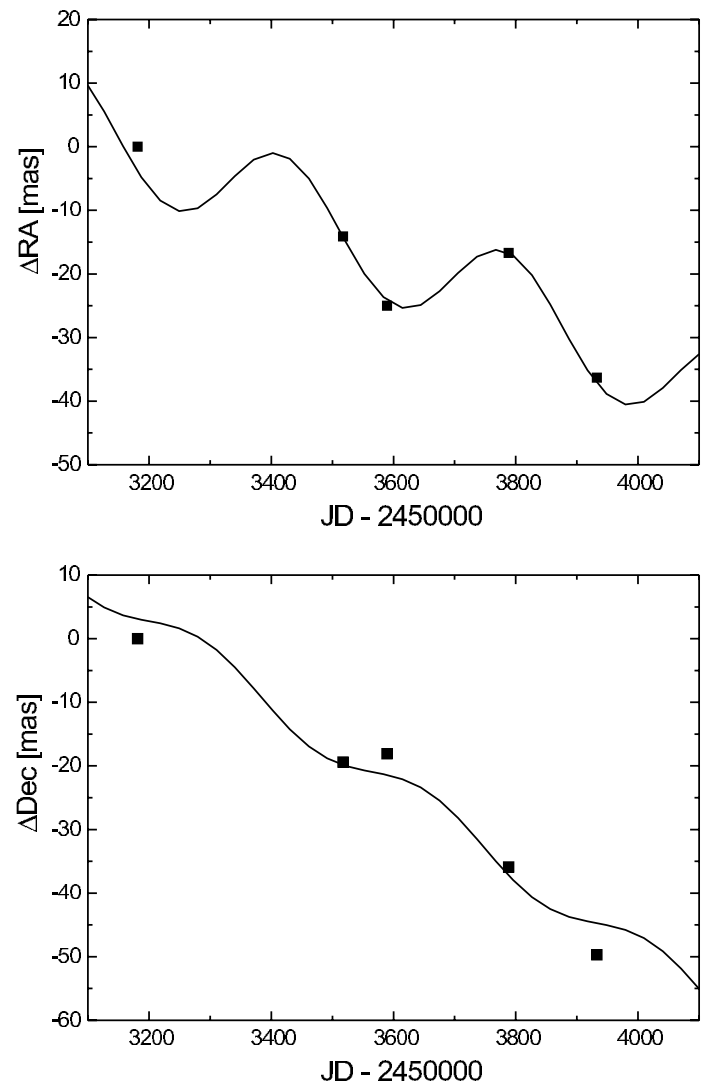

Fig. 4. Proper and parallactic motion of GQ Lupi b in right ascension (top) and declination (bottom) relative to the background object GQ Lupi/cc2. Changes in separations in mas (since 2004.5) are plotted versus observing epoch as Julian date JD. We also show our best fit yielding a parallactic wobble of $8.2 \pm 2.1$ mas, from which we have to subtract the probable parallax of the background object cc2 $(1.0 \pm 0.3$ mas, see text), thus obtaining $7.2 \pm 2.1$ mas or $139 \pm 45 \mathrm{pc}$ for the GQ Lupi companion.

comparing to this comparison star shows that its proper motion is very small.

The error in the parallax determination is quite large, which is due to the following reasons.

(i) We use only one comparison star.

(ii) The GQ Lupi companion and the comparison star are quite faint.

(iii) There are statical abberrations and field distortions of the CONICA S13 camera amounting to up to 3 mas at its edges ${ }^{3}$. Since we measure relative changes between different epochs, not absolute positions, the important question for a parallax measurement is the repeatability of the configuration between the epochs. Since the jitter/dither pattern at each epoch moves the reference line (GQ Lupi to field star) over the FoV, the variations in this separation from the field distortion are dominating the uncertainties in the angle and length of the reference line in each epoch and are

\footnotetext{
3 We are monitoring these field distortions in a different (astrometric) observing program, where we observe the 47 Tuc cluster center several times per year with the NACO S13 FoV and always the same set-up (also 2005 to 2007 as GQ Lupi). We achieved sub-mas relative precision; first results are published in Neuhäuser et al. (2007) and Seifahrt et al. (2007a), more details will be given in Roell et al. (in preparation). From these observations, we already know that the field distortions are very small except at the field edges.
}

Table 4. Photometry on GQ Lupi with VLT/NACO.

\begin{tabular}{lcccc}
\hline \hline Epoch & Diff. (a) & \multicolumn{3}{c}{$K_{\mathrm{s}}$ band magnitude [mag] } \\
year & HIP A-B & GQ Lupi A & GQ Lupi b & cc2 (b) \\
\hline 2005.40 & $1.56 \pm 0.07$ & $7.07 \pm 0.07$ & $13.46 \pm 0.12$ & $14.80 \pm 0.24$ \\
2005.60 & $1.46 \pm 0.13$ & $7.04 \pm 0.14$ & $13.34 \pm 0.42$ & $14.78 \pm 0.18$ \\
2006.14 & $1.57 \pm 0.08$ & $7.09 \pm 0.08$ & $13.38 \pm 0.10$ & $14.88 \pm 0.16$ \\
2006.38 & $1.59 \pm 0.07$ & $7.40 \pm 0.08$ & $13.47 \pm 0.14$ & $14.87 \pm 0.26$ \\
2006.54 & $1.66 \pm 0.12$ & $7.02 \pm 0.13$ & $13.45 \pm 0.38$ & $15.07 \pm 0.34$ \\
2007.13 & $1.54 \pm 0.16$ & $7.48 \pm 0.16$ & $13.27 \pm 0.12$ & $15.67 \pm 0.92$ \\
\hline mean & $1.56 \pm 0.07$ & $7.18 \pm 0.20$ & $13.39 \pm 0.08$ & $15.01 \pm 0.33$ \\
\hline
\end{tabular}

Remarks: (a) difference in $K_{\mathrm{s}}$ between HIP 73357 A and B. (b) background object GQ Lupi/cc2.

contained in the error budget. Since we have always chosen a small jitter/dither box/offset, GQ Lupi and its very nearby companion are always located near the center of the FoV and the comparison star is also always located nearly at the same spot on the detector (within few arcsec), so that the distortions seen by the camera are always the same between the epochs. Since this is a relative measurement, not an absolute one, the remaining distortion term affecting the true length and angle of the reference line is not important here.

(iv) Differential chromatic refraction has a negligible effect in the $K$ band. The wavelength dependence of the refractive index of air is very low in $K$, and the differential terms from the color difference of GQ Lupi and the background star are much smaller (both are late K-type dwarfs), because we operate in the Rayleigh-Jeans term of the spectral energy distribution of both targets. The remaining effect is much smaller than 0.5 mas.

(v) Differential AO correction is certainly an issue when comparing GQ Lupi or its companion to the comparison star, because it is located several arcsec away, while GQ Lupi and its companion, within 1 arcsec, are inside the isoplanatic angle.

Effects (i), (ii), and (v) are largest and lead to the relatively large error bar. However, the parallactic wobble effect is still clearly seen in Figs. 3 and 4.

What we measure is the difference between the GQ Lupi parallax and the parallax of this comparison star. If we further assume that it is a background star at large distance with negligible parallax, then we can interpret the measurement as the parallax of GQ Lupi. We can also subtract the probable parallax of the background star $(1.0 \pm 0.3$ mas, see above) from the total difference in parallax measured here, thus obtaining $6.4 \pm 1.9$ mas as parallax of GQ Lupi A (i.e. $156 \pm 50 \mathrm{pc}$ ) and also $7.2 \pm 2.1 \mathrm{mas}$ as parallax of GQ Lupi b (i.e. $139 \pm 45 \mathrm{pc}$ ). These values are not more precise than the original assumption $(140 \pm 50 \mathrm{pc}$, N05 for the Lupus I cloud), but our measurements show that GQ Lupi A and its companion are located at the same distance and at the same distance as the Lupus I cloud and its members.

Given these uncertainties and limitations, our measurement needs to be confirmed. If correct, it may be the smallest parallax determined from the ground (but with large error). This method can also be applied to other suitable targets, which have a background object within the small AO FoV.

\section{Photometry}

During the service mode observations of GQ Lupi, it was either clear at least in and around Lupus and around HIP 73357 


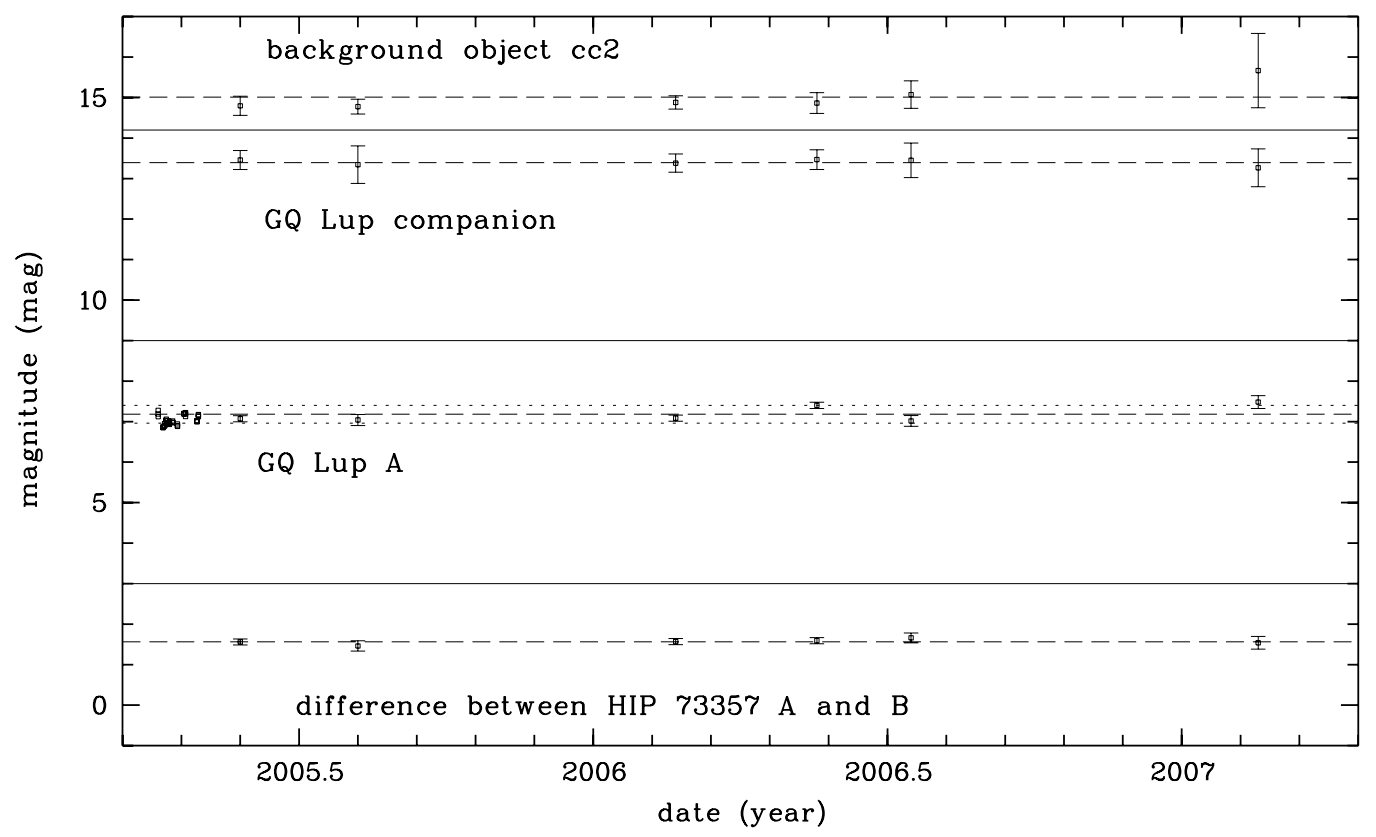

Fig. 5. $K_{\mathrm{s}}$ band magnitude versus time for difference between HIP 73357 A and B (bottom panel), GQ Lupi A (2nd from bottom), GQ Lupi companion (2nd from top), and the background object GQ Lupi/cc2 (top panel). We always show as a long-dashed line the mean value. In the case of GQ Lupi A, we also show the data points from Broeg et al. (2007), for which absolute photometry is available (C. Broeg, priv. comm.) as small dots in April 2005; we also show as dotted lines the range in variability ( $\pm 0.22 \mathrm{mag}$ ) found by Broeg et al. (2007) during their monitoring of GQ Lupi A (yielding the rotation period to be $\sim 8.4$ days). We find variability in GQ Lupi A (amplitude \pm 0.20 mag, $4 \sigma$ ).

or photometric; HIP 73357 was always observed either immediately before or after GQ Lupi. After aperture photometry on HIP $73357 \mathrm{~A}$ and $\mathrm{B}$, we notice that the difference between the $K_{\mathrm{s}}$-band magnitudes of $\mathrm{A}$ and $\mathrm{B}$ does not vary with time (see Fig. 5 bottom panel). Hence, they can both be assumed to be constant (they are neither known nor expected to be variable). We can then use their 2MASS magnitudes $\left(K_{\mathrm{S}}=6.467 \pm 0.016 \mathrm{mag}\right.$ for HIP $73357 \mathrm{~A}$ and $K_{\mathrm{s}}=8.027 \pm 0.021 \mathrm{mag}$ for HIP $73357 \mathrm{~B}$ ) and the respective airmasses to correct the instrumental magnitudes obtained for the objects in the GQ Lupi field. Aperture photometry was performed in the same way on GQ Lupi A, its companion (after subtraction of PSF of GQ Lupi A), and the background object cc2. See Table 4 for the $K_{\mathrm{s}}$-band magnitudes. The error budget includes the 2MASS magnitude errors of HIP 73357 A and B, the measurement errors in aperture photometry, airmass, and extinction coefficient. We display the variation of $K_{\mathrm{s}}$ band magnitudes with time in Fig. 5 for the difference between HIP 73357 A and B, GQ Lupi A, its companion b, and the background object cc2.

The mean magnitude of GQ Lupi A (Table 4) is consistent with its 2 MASS value $\left(K_{\mathrm{s}}=7.096 \pm 0.020 \mathrm{mag}\right)$, obtained at an unknown rotation phase. We confirm the variability of GQ Lupi A in the $K_{\mathrm{s}}$-band ( $4 \sigma$ significance) already found by Broeg et al. (2007), interpreted as being due to surface spots and a $\sim 8.4$ day rotation period. Broeg et al. (2007) found an amplitude in $K_{\mathrm{S}}$ in April/May 2005 of $\pm 0.22 \mathrm{mag}$; we obtain \pm 0.20 for May 2005 to Feb. 2007. In our data from May 2005 to Feb. 2007, GQ Lupi A varies between 7.02 and 7.48 mag (Feb. 2007), while it varies between 6.85 and $7.28 \mathrm{mag}$ in April 2005 (Broeg et al. 2007 and C. Broeg, private communication). There may be a slight dimming of GQ Lupi A with time, possibly due to temporal changes in the spottedness.

The mean magnitude of the GQ Lupi companion given here (Table 4) is slightly fainter than given in N05 $(13.10 \pm 0.15 \mathrm{mag}$ for June 2004), but within $2 \sigma$ errors, due to the fact that we have now improved on the subtraction of the GQ Lupi A PSF (see above). The new mean value is consistent with the value given by Marois et al. (2007) for the 2002 Subaru exposure $\left(K_{\mathrm{s}}=\right.$ $13.37 \pm 0.12 \mathrm{mag})$. Given its faintness and the large error bars, we cannot find evidence for variability of the GQ Lupi companion in the $K_{\mathrm{s}}$-band. The $K_{\mathrm{s}}$-band values show a small standard deviation of \pm 0.08 mag, which could be due to variability in the GQ Lupi companion and/or HIP 73357; possible variations would be expected to have a smaller amplitude than in GQ Lupi A, just because the companion is much fainter. Variability would be expected, given that it is a young object ( $\leq 2$ Myr as GQ Lupi A) and that Paschen $\beta$ was found to be in emission by Seifahrt et al. (2007a), a sign of ongoing accretion.

\section{Deep imaging}

We use all imaging data obtained from June 2004 to July 2006 (see Table 1, i.e., omitting the data from February 2007 because of low quality, e.g., strong reflections and waffle structure) to combine all of them together by shift+add (as usual with ESO eclipse) to get a very deep image with very high dynamic range. We use the IDL routine for PSF subtraction as explained above to subtract the PSF of GQ Lupi A. The resulting image after PSF subtraction is shown in Fig. 6. We can then measure the background level in all pixels and at all separations from the (former) photocenter of GQ Lupi A.

The flux ratio between the peak of GQ Lupi A (before PSF subtraction) and the background (in the PSF-subtracted image) versus the separation is then plotted in Fig. 7 and compared to the dynamic range before PSF subtraction. No further companion candidates are detected. We can exclude companion candidates with at least the flux of GQ Lupi b outside of $\sim 0.115^{\prime \prime}$ or $\sim 18 \mathrm{AU}$ (at $156 \mathrm{pc}$ ), or outside of $23 \mathrm{AU}$ without PSF subtraction. Closer companions (or those located before or behind GQ Lupi A) cannot be excluded from the imaging alone. As displayed in Fig. 7 , 


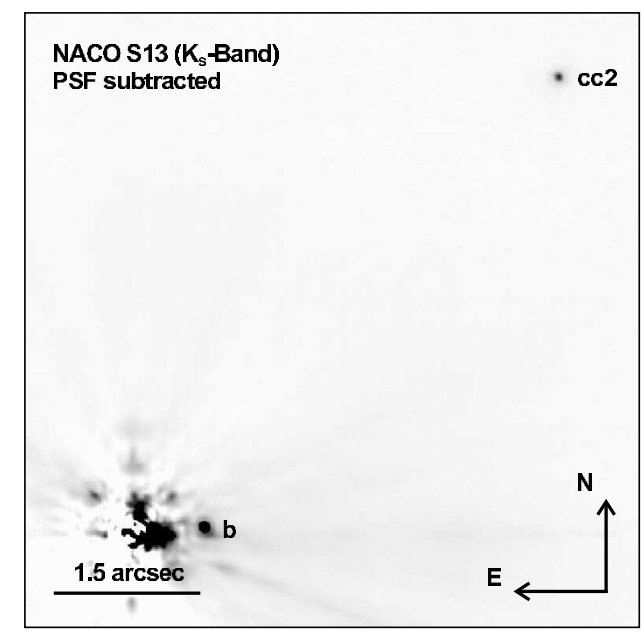

Fig. 6. Deepest image of GQ Lupi (102 min total exposure) after subtraction of PSF of GQ Lupi A. North is up, east to the left. The companion GQ Lupi b is seen well 0.7" west of GQ Lupi A, whose PSF was subtracted. The background object GQ Lupi/cc2 is seen in the upper right $(\mathrm{NW})$ corner.

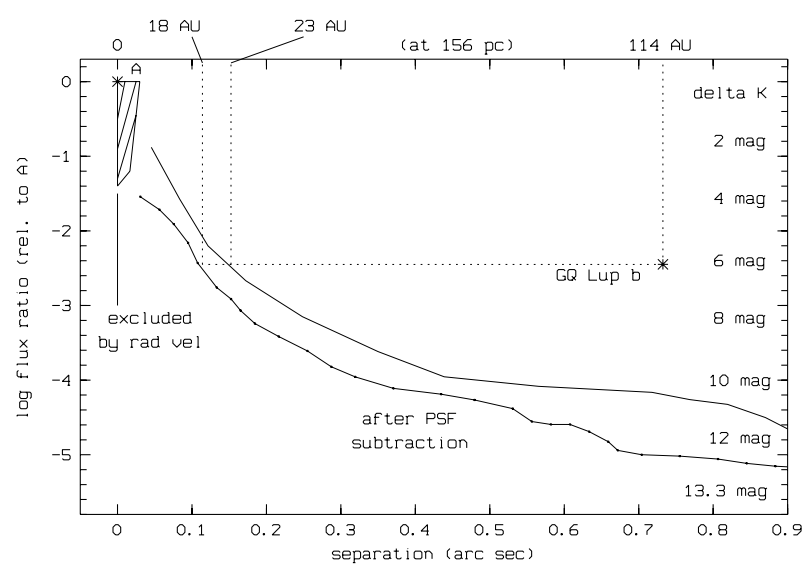

Fig. 7. Dynamic range from deepest image shown in Fig. 6. We plot ( $\log$ of) noise flux $(S / N=3)$ devided by the peak flux of GQ Lupi A (before PSF subtraction) on the left axis (and the magnitude difference at the right axis) versus separation in arcsec on the bottom axis (versus separation in AU at $156 \mathrm{pc}$ on the top axis). The lower full line with points is obtained after PSF subtraction, the upper line is without PSF subtraction. GQ Lupi and its companion are indicated as star symbols. The hatched area in the upper left is the region in this parameter space, where companions can be excluded by high-resolution spectroscopic monitoring of the radial velocity (rad vel) by Broeg et al. (2007), see text.

we would be able to detect companions, e.g., $\leq 10$ mag fainter (in $K_{\mathrm{s}}$ ) than GQ Lupi A at $\geq 0.3^{\prime \prime}$ separation or $\leq 12$ mag fainter at $\geq 0.7^{\prime \prime}$ (after PSF subtraction). Between about 0.6" and 0.9", we could gain $\sim 1$ mag dynamic range by PSF subtraction.

Broeg et al. (2007) have monitored GQ Lupi for several years with the ESO high-resolution spectrographs FEROS and HARPS and searched for companions with the radial velocity method; they could not detect any spectroscopic companions with mass above $\sim 0.1 M_{\odot}$ inside $\sim 2.6 \mathrm{AU}$. With $L_{\text {bol }} \simeq 0.1 L_{\odot}$ for a few Myr young $\sim 0.1 M_{\odot}$ star (Burrows et al. 1997) and using $L_{\text {bol }}=1.58 L_{\odot}$ for GQ Lupi A (N05), we can also display the area in Fig. 7, where companions can be excluded by the spectroscopic monitoring. This area is complementary to the area investigated by the imaging.
Debes \& Sigurdsson (2006) and Boss (2006) suggested that GQ Lupi b, if a planet, would have been ejected by a near encounter with another protoplanet, which would most likely be located within 10 AU of GQ Lupi A and would need to be more massive than GQ Lupi b. We could not detect such a close-in massive planet, yet. The radial velocity monitoring of GQ Lupi A (Broeg et al. 2007) also could not yet detect a closein massive planet. However, there is still a large separation range not yet probed with either imaging or spectroscopy (between few and $18 \mathrm{AU})$, where further companions can hide.

\section{Summary of results and conclusions}

Given the newly constrained mean $K_{\mathrm{s}}$-band magnitude of $13.39 \pm$ $0.08 \mathrm{mag}$ and distance measured for the companion of $139 \pm$ $45 \mathrm{pc}$ (this paper) and with B.C. $\mathrm{K}=3.0 \pm 0.1 \mathrm{mag}$ (following Golimowski et al. 2004) and the temperature of the companion newly constrained in Seifahrt et al. (2007a), we can re-estimate the luminosity of the companion to be $\log \left(L_{\text {bol }} / L_{\odot}\right)=-2.375 \pm$ 0.245 , similar to the value in N05, but with a smaller error bar.

With the temperature $2650 \pm 100 \mathrm{~K}$ and gravity $\log g=3.7 \pm$ $0.5 \mathrm{dex}\left(\mathrm{g}\right.$ in $\left.\mathrm{g} / \mathrm{cm}^{2}\right)$ for the GQ Lupi companion (Seifahrt et al. 2007a), we can use luminosity and temperature to re-calculate its radius to be $3.0 \pm 0.5 R_{\mathrm{Jup}}$. With radius and gravity, we obtain a mass of $\sim 20 M_{\text {Jup }}$ with a possible minimum (value $-1 \sigma$ ) being only few $M_{\mathrm{Jup}}$, and the maximum being around the sub-stellar limit. However, the upper mass limit for the GQ Lupi companion is still $36 \pm 3 M_{\text {Jup }}$, because the GQ Lupi companion is smaller, cooler, and fainter than both components in the eclipsing doublelined spectroscopic binary brown dwarf 2M0535 (Stassun et al. 2006), which has a similar age as GQ Lupi, as already noticed by Seifahrt et al. (2007a).

We can also use luminosity $L$, temperature $T$, gravity $g$, radius $R$, and the age of the young T Tauri star GQ Lupi ( $\leq 2 \mathrm{Myr}$, N05, having strong IR excess) to estimate the mass of the companion from theoretical evolutionary models (as done in N05 with the original, less constrained parameters): from Burrows et al. (1997), we consistently obtain for all combinations of $L$, $T, g, R$, and age a mass of $\sim 20 M_{\text {Jup }}$, and from Baraffe et al. (2002), we consistently get for all combinations $L, T$, and age a mass of $\sim 20 M_{\text {Jup }}$. According to the calculations following Wuchterl \& Tscharnuter (2003), as plotted in Fig. 4 in N05, the GQ Lupi companion would have $\sim 5 M_{\text {Jup }}$. It may be seen as intriguing that both the atmospheric and the conventional evolutionary models consistently give $\sim 20 M_{\text {Jup }}$ as the best value. However, we note that the models by Burrows et al. and Baraffe et al. may not be valid for very young objects ( $\leq 10 \mathrm{Myr})$, as initial conditions are not taken into account, and none of the models used are tested positively for very low-mass objects or calibrated.

A better mass estimate can be obtained in the future by comparison with more very young objects with dynamically determined masses and/or atmospheric or evolutionary models that are calibrated.

We can summarize our results as follows.

1. With precise relative astrometry at six new epochs, we have detected small deviations in the separation between GQ Lupi A and its companion, consistent with orbital motion of $\sim 2$ to 3 mas/yr.

2. It remains unclear whether the slight decrease in separation observed is due to orbital motion around each other (bound) or due to different parallaxes (unbound). The latter case is, however, less likely. 
3. By comparing the position of GQ Lupi A and its companion to a third object in the small NACO FoV, a background star at 800 to $1700 \mathrm{pc}$, we could determine the parallaxes of GQ Lupi A and its companion corresponding to a distance of $156 \pm 50 \mathrm{pc}$ for A and $139 \pm 45 \mathrm{pc}$ for the companion.

4. Apart from that background star, no additional companion candidates are detected. Outside of 115 mas (18 AU), we can exclude further companion candidates as bright or brighter than the known co-moving companion.

5. We could confirm photometric variability of GQ Lupi A in the $K_{\mathrm{s}}$-band ( 7 exposures over 3 years); variability of the companion is smaller than $\pm 0.08 \mathrm{mag}$.

6. From the newly constrained distance and the mean $K_{\mathrm{s}^{-}}$ band magnitude, we re-estimate luminosity, radius, and mass of the companion, but we can still not decide whether the companion is below the deuterium burning mass limit (or below the radial velocity brown dwarf desert, which is at $\sim 30 M_{\text {Jup }}$ according to Grether \& Lineweaver 2006), i.e., whether the companion is of planetary mass or a brown dwarf. The large uncertainty in mass is due to uncertainties in the theoretical models and the gravity and distance measurements.

Acknowledgements. We are gratefull to ESO's User Support Department for help in preparation of the service mode observations and carrying out our observations. TOBS would like to thank Evangelisches Studienwerk e.V. Villigst for financial support. N.V. acknowledges support by FONDECYT grant 1061199. We would like to thank Christopher Broeg for providing his photometric data on GQ Lupi A in electronic form.

\section{Appendix A: Astrometric calibration}

For the astrometric calibration, we observed from 2005 to 2007 always the same binary star HIP 73357, where the Hipparcos satellite measured the separation to be $8.43 \pm 0.03^{\prime \prime}$ and the position angle (PA, measured from north over east to south) to be $337.30 \pm 0.06^{\circ}$ (at epoch 1991.25). HIP $73357 \mathrm{~A}$ and $\mathrm{B}$ have spectral types mid-A and mid-F, hence masses of $\sim 2.5$ and $\sim 1.5 M_{\odot}$, and a distance of $104 \pm 22 \mathrm{pc}$. This results in an orbital period of $\sim 13000$ years (for circular orbit, shorter if eccentric) and, hence, a maximum orbital motion of $\sim 2.5$ mas/yr for edgeon inclination and $\sim 0.025^{\circ} / \mathrm{yr}$ for face-on inclination (both for an eccentricity of $e \simeq 0.2)^{4}$. It is not confirmed that HIP 73357 A and $\mathrm{B}$ form a bound pair orbiting each other (curvature in orbital motion is not yet detected). Given their known (and very similar) proper motions (they form a common-proper-motion pair), the motion of A relative to B results in slightly less changes in separation and PA than the maximum orbital motion given above.

Absolute values and their errors for separation and PA measured for HIP 73357 and GQ Lupi images include the errors in separation and PA for HIP 73357 AB from Hipparcos, errors from Gaussian centering fits on HIP 73357 A and B, and GQ Lupi A and companion, as well as the maximum possible (orbital) motion (of HIP 73357 A relative to B) between 1991.25 and the new observation at each epoch. For absolute errors (given in Table 1) we include the maximum (orbital) motion from 1991.25 to the new epoch. Values for changes in separation and PA with their (relative) errors (as given in Table 1) include only the maximum relative (orbital) motion since the first new

\footnotetext{
4 We confirm a-posteriori, see below, that these assumptions are probably correct. In reality, we have started with slightly other assumptions and have then iterated; in the iterations, the values for pixel scale and detector orientations stayed constant, just the (maximal) errors due to (maximal) orbital motion changed.
}

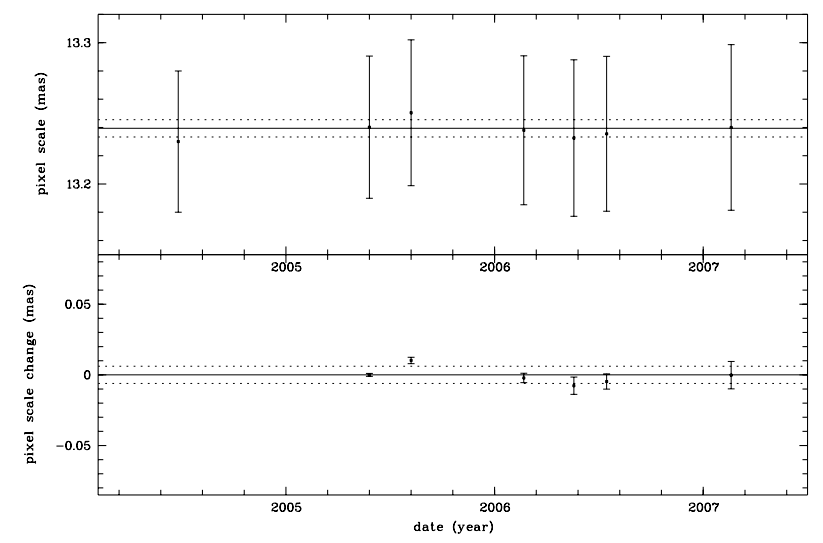

Fig. A.1. NACO pixel scales as determined from binary HIP 73357 observed within one hour from GQ Lupi (but see footnote 1 for Feb. 2007). We show absolute values and errors in the upper panel (including errors due to (orbital) motion in HIP 73357 since Hipparcos at epoch 1991.25) and relative values for pixel scale changes with relative errors in the bottom panel (including errors due to (orbital) motion in HIP 73357 only since our first new epoch 2005.4), and also showing the data point at the first new epoch 2005.4, set to 0.0, with its error bar just from the Gaussian centering fit. Both panels show the same range in pixel scale on the $y$-axis, 0.17 mas in both panels.

epoch (2005.4, we observed a different astrometric calibration binary in 2004) and, of course, also the errors from Gaussian centering fits. The relative errors are smaller than the absolute errors. This way, we can obtain better precision to detect orbital motion in GQ Lupi as changes in separation and/or PA, but we cannot give anymore the absolute values for separation (in arc$\mathrm{sec}$ ) or PA on sky (in degrees), but can measure their changes.

The detector orientations given in Table 1 are the difference between the observed PA and $337.3^{\circ}$ (from Hipparcos at 1991.25). To obtain the true PA of, e.g., the GQ Lupi companion, we have to add the PA value measured on GQ Lupi images with the value given for the detector orientation. Such an absolute value for the PA (similar for pixel scale and separation) can also be given with (absolute) errors (accuracy). The relative errors are not applicable to the absolute PA values, but only to PA changes since 2005.4 (relative errors for precision).

The values for separation and PA at 2005.4 have also their own measurement errors (from Gaussian centering only, without uncertainty in (orbital) motion of HIP 73357), also listed in Table 2 under relative error.

We display the pixel scale and detector orientation values for all epochs in Figs. A.1 and A.2. The mean pixel scale is $13.2394 \pm 0.0061 \mathrm{mas} /$ pixel with 0.054 mas as the mean absolute error bar and 0.0031 mas as the mean relative error. The detector orientation has a mean of $0.29 \pm 0.11^{\circ}$; for orientation changes since May 2005, the mean is $0.09 \pm 0.11^{\circ}$ (and $0.024^{\circ}$ as mean relative error). These values may indicate that HIP 73357 $\mathrm{A}$ and B show an orbital motion of $\sim 0.3^{\circ}$ since 1991.25 (similar as assumed, see footnote 4), but we would need absolute astrometric reference objects as a confirmation. If true, the precision of the NACO detector alignment during target acquisition is around \pm 0.1 degree. Fitting the seven data points shown in Fig. A.2 (upper panel) with a constant line without slope (mean detector orientation) gives a similarily good fit (i.e. larger rms, reduced $\chi^{2}=0.85$ ) as fitting it to a detector orientation increasing constantly from $0^{\circ}$ at 1991.25 to the mean value 0.29 observed at the mean of the new observing epochs (2005.9536), as drawn in Fig. A.2 (reduced $\chi^{2}=0.97$ ). The pixel scales determined do not show any slope (Fig. A.1). 


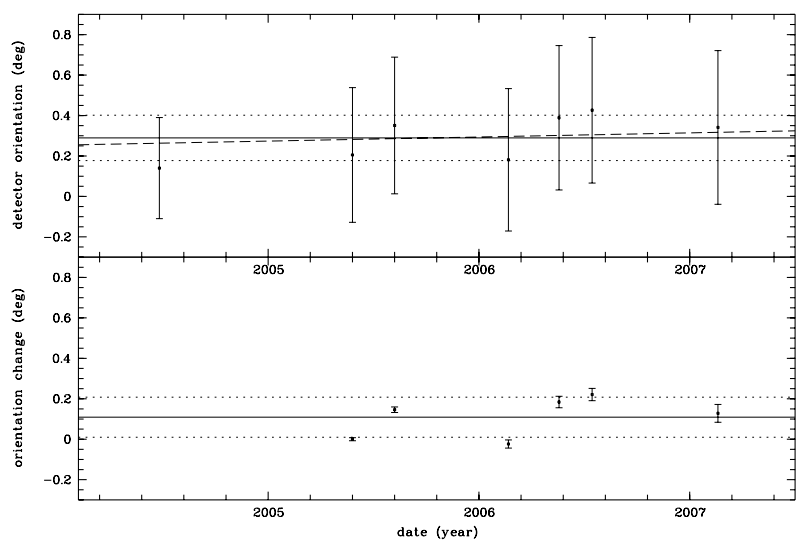

Fig. A.2. NACO detector orientations as determined from binary HIP 73357 observed within one hour from GQ Lupi (but see footnote 1 for Feb. 2007). Upper and lower panels are as in Fig. A.1. The dashed line in the upper panel shows the best fit to the data consistent with a detector orientation of $0^{\circ}$ at 1991.25, the Hipparcos epoch, indicating that the small increase in detector orientation with time can be interpreted as orbital motion in HIP 73357 A and B. Both panels show the same range in $\mathrm{PA}$ on the $y$-axis, $1.2^{\circ}$ in both panels.

The observed orbital motion in HIP 73357 is near the maximum orbital motion estimated above $\left(\sim 0.39^{\circ}\right.$ from 1991.25 to 2005.4), therefore (i) the orbital inclination may well be near pole-on, (ii) the eccentricity near $\sim 0.2$, and (iii) the change in separation should be negligible, which we see in the roughly constant pixel scale obtained by assuming that the separation between HIP 73357 A and B is constant.

The large change in PA in HIP 73357 results in larger errors in the PA calibration, while the errors in pixel scale calibration remain very small, because the separation remains (nearly) constant; absolute PA errors are always larger than $0.3^{\circ}$, the offset between the Hipparcos value and as observed with NACO, the difference between $0.3^{\circ}$ and the PA errors given in Table 2 (even at epoch 2005.4) are due to Gaussian centering errors; errors in both PA and pixel scale - with few exceptions - increase with time due to additional possible orbital motion.

The stars HIP $73357 \mathrm{~A}$ and B are located near the edges of the small S13 NACO FoV, and the jitter offsets are relatively large in right ascension, so that field distortions may play an important role. In an attempt to check the errors of our astrometry, we not only measured separations and orientations in one full final high- $S / N$ image after combining all images of one epoch (one night), but we also measured separation and PA between HIP 73357 A and B (and similar for GQ Lupi), in any of the five HIP 73357 images (43 good $S / N$ images of HIP 73357 for Feb. 2007, see footnote 1). We can then take the mean of the separation and PA values and their standard deviation as alternative measurement. They are in agreement with the standard measurement (obtained after combining all five images of HIP 73357) within small errors. In the case of HIP 73357, the errors of these two measurement methods are comparable. To be conservative, we always use the larger error value for absolute errors (as listed in Tables 1 and 2).

\section{References}

Baraffe, I., Chabrier, G., Allard, F., \& Hauschildt, P. H. 2002, A\&A, 382, 563 Boss, A. P. 2006, ApJ, 637, L137

Broeg, C., Schmidt, T. O. B., Guenther, E. W., et al. 2007, A\&A, 468, 1039 Burrows, A., Marley, M., Hubbard, W., et al. 1997, ApJ, 491, 856

Chabrier, G., Baraffe, I., Allard, F., \& Hauschildt, P. H. 2005, Review on lowmass stars and brown dwarfs, in: Resolved Stellar Populations Conf. Proc. [arXiv: astro-ph/0509798]

Debes, J. H., \& Sigurdsson, S. 2006, A\&A, 451, 351

Golimowski, D. A., Leggett, S., Marley, M., et al. 2004, AJ, 127, 3516

Janson, M., Brandner, W., Henning, T., \& Zinnecker, H. 2006, A\&A, 453, 609 Grether, D., \& Lineweaver, C. H. 2006, ApJ, 640, 1051

Kirkpatrick, J. D., Barman, T. S., Burgasser, A. J., et al. 2006, ApJ, 639, 1120 Marois, C., Macintosh, B., \& Barman, T. 2007, ApJ, 654, L151

McElwain, M. W., Metchev, S. A., Larkin, J. E., et al. 2007, ApJ, 656, 505

Mugrauer, M., \& Neuhäuser, R. 2005, AN, 361, L15

Neuhäuser, R., Guenther, E. W., Wuchterl, G., et al. 2005, A\&A, 435, L13 (N05)

Neuhäuser, R., Seifahrt, A., Roell, T., Bedalov, A., \& Mugrauer, M. 2007, Detectability of Planets in Wide Binaries by Ground-Based Relative Astrometry with AO, in: Binary Stars as Critical Tools and Tests in Contemporary Astrophysics, ed. W. I. Hartkopf, E. F. Guinan, \& Harmanec, IAU Symp. 240, 261

Rousset, G., Lacombe, F., Puget, P., et al. 2003, SPIE, 4839, 140

Seifahrt, A., Neuhäuser, R., \& Hauschildt, P. H. 2007a, A\&A, 463, 309

Seifahrt, A., Roell, T., \& Neuhäuser, R. 2007b, Probing micro-arcsec astrometry with NACO, in Proceedings of the 2007 ESO Instrument Calibration Workshop, in press [arXiv: astro-ph/0706.2613]

Stassun, K. G., Mathieu, R. D., \& Valenti, J. A. 2006, Nature, 440, 311

Wuchterl, G., \& Tscharnuter, W. M. 2003, A\&A, 398, 1081 